Pacific Journal of Mathematics

ON NON-ASSOCIATIVE ALGEBRAS ASSOCIATED WITH 


\section{ON NON-ASSOCIATIVE ALGEBRAS ASSOCIATED WITH BILINEAR FORMS}

\section{W. E. JENNER}

If $\mathfrak{B}_{0}$ is a vector space over a field $k$, then with any non-degenerate bilinear form $f_{0}$ on $\mathfrak{S}_{0} \times \mathfrak{B}_{0}$ is associated the group (B) of linear transformations of $\mathfrak{B}_{0}$ which keep $f_{0}$ invariant. In this paper a procedure is given for associating with such a bilinear form an algebra $\mathfrak{A}$, nonassociative in general, whose automorphism group is isomorphic to (S) and which is right and left simple provided $\mathfrak{B}_{0}$ has dimension at least 2. In case $k$ is the field of real numbers, then (B) is a Lie group and its Lie algebra is the Lie algebra of derivations of $\mathfrak{A}$. In case the form $f_{0}$ is degenerate, and either symmetric or alternating, then the analogue of the Wedderburn Principal Theorem holds for $\mathfrak{A}$. The results obtained apply, in particular, to the orthogonal and symplectic groups.

Let $\mathfrak{B}_{0}$ be a vector space of dimension $n$ over a field $k$ with basis $u_{1}, \cdots, u_{n}$. It is assumed that $\lambda v=v \lambda$ for all $v \in \mathfrak{S}_{0}$ and $\lambda \in k$. Suppose $f_{0}$ is a bilinear form on $\mathfrak{B}_{0} \times \mathfrak{B}_{0}$. Define $\mathfrak{A}$ to be the algebra over $k$ with basis $e_{0}, e_{1}, \cdots, e_{n}$ and multiplication table $e_{0}^{2}=e_{0}, e_{i} \cdot e_{0}=e_{0} \cdot$ $e_{i}=e_{i}, e_{i} \cdot e_{j}=f\left(e_{i} e_{j}\right) e_{0}$ for $i, j=1,2, \cdots, n$, where $f\left(e_{i}, e_{j}\right)=f_{0}\left(u_{i}, u_{j}\right)$. Let $\mathfrak{B}$ be the subspace of $\mathfrak{A}$ spanned be $e_{1}, \cdots, e_{n}$. Then $f$ is a bilinear form on $\mathfrak{B} \times \mathfrak{B}$.

THEOREM 1. Suppose that $f$ is non-degenerate and that $n \geqq 2$. Then $\mathfrak{A}$ is right and left simple.

Proof. Let $\mathfrak{U}$ be a non-zero left ideal of $\mathfrak{A}$ and let $u$ be a non-zero element of $\mathfrak{U}$. Suppose first that $u \in \mathfrak{B}$. Then there exists an element $v \in V$ such that $f(v, u) \neq 0$. Then $v \cdot u=f(v, u) e_{0}$. Therefore $e_{0} \in \mathfrak{u}$ and so $\mathfrak{U}=\mathfrak{A}$. Next suppose $u=\alpha e_{0}+v$ where $\alpha \neq 0$ in $k$ and $v \in V$. Then one can assume $\alpha=1$. Since $n \geqq 2$ it follows that $e_{1} \cdot u=e_{1}+\lambda_{1} e_{0}$ and $e_{2} \cdot u=e_{2}+\lambda_{2} e_{0}$ where $\lambda_{1}, \lambda_{2} \in k$. If $\lambda_{1}=0$ then $e_{1} \in U$ and the first part of the proof applies; similarly if $\lambda_{2}=0$. Consequently one can suppose $\lambda_{1} \lambda_{2} \neq 0$. Then $\lambda_{2} e_{1} u-\lambda_{1} e_{2} u=\lambda_{2} e_{1}-\lambda_{1} e_{2}$ is a non-zero element in $\mathfrak{U} \cap \mathfrak{B}$. Thus the first part of the proof again applies and so $\mathfrak{U}=\mathfrak{A}$. Therefore $\mathfrak{A}$ is left simple; similarly $\mathfrak{A}$ is right simple.

If $\mathfrak{A}$ is any (non-associative) algebra over $k$ then left (right) multiplication by an element $a \in \mathfrak{A}$ determines a linear transformation $L_{a}\left(R_{a}\right)$ of the underlying vector space of $\mathfrak{A}$ by $a \cdot u=L_{a} u\left(u \cdot a=R_{a} u\right), u \in \mathfrak{A}$. The set of linear transformations $L_{a}\left(R_{a}\right)$ for $a \in \mathfrak{A}$ generate an associative algebra $L(\mathfrak{U})(R(\mathfrak{H}))$ over $k$. The algebras $L(\mathfrak{U})$ and $R(\mathfrak{U})$ together

Received May 8, 1959. 
generate the transformation algebra $T(\mathfrak{A})$.

THEOREM 2. If $f$ is non-degenerate and $n \geqq 2$ then $L(\mathfrak{U})=R(\mathfrak{H})=$ $T(\mathfrak{U})=[k]_{n+1}$.

Proof. The proof of Theorem 1 shows that for any $u \neq 0$ in $\mathfrak{A}$ there is an element of $L(\mathfrak{U})$ mapping $u$ into any arbitrarily assigned element of $\mathfrak{A}$. Therefore $L(\mathfrak{U})=[k]_{n+1}$; similarly for $R(\mathfrak{U})$, and so also for $T(\mathfrak{X})$.

Albert has introduced in [1] the concept of isotopy of non-associative algebras. Suppose $\mathfrak{A}$ is an algebra with left multiplications $L_{a}$ defined by $a \cdot u=L_{a} u$. Then an isotope of $\mathfrak{A}$ is an algebra $\mathfrak{X}^{0}$ with the same underlying vector space and multiplication defined by $a \circ u=P L_{a Q} S u$ where $P, Q, S$ are invertible linear transformations of the underlying vector space of $\mathfrak{A}$. An algebra $\mathfrak{A}$ is said to be isotopically left (right) simple if every isotope of $\mathfrak{A}$ is left (right) simple.

THeOREM 3. Suppose $f$ is non-degenerate and that $n \geqq 2$. Then $\mathfrak{A}$ is isotopically left and right simple.

Proof. Suppose $\mathfrak{U}$ is a subspace of $\mathfrak{U}$ such that $P L_{x Q} S \mathfrak{u} \subseteq \mathfrak{U}$ for all $x \in \mathfrak{A}$. Now choose $x \in \mathfrak{U}$ such that $L_{x Q}=L_{e_{0}}=I$, the identity transformation. Then $P S \mathfrak{U} \subseteq \mathfrak{U}$. Therefore $P S \mathfrak{U}=\mathfrak{U}$ and $S \mathfrak{U}=P^{-1} \mathfrak{d}$ since $P$ and $S$ are invertible. Then for any $u \in \mathfrak{A}, L_{u Q} S \mathfrak{U} \subseteq P^{-1} \mathfrak{U}=S \mathfrak{U}$ and so $S \mathfrak{U}$ is a left ideal of $\mathfrak{U}$. Therefore either $\mathfrak{U}=(0)$ or $\mathfrak{A}$. Consequently $\mathfrak{A}$ is isotopically left simple; similarly it is isotopically right simple.

REMARK. Bruck has shown in [2] that left and right isotopic simplicity follow from left and right simplicity if the algebra has a unit element. The proof has been given here for sake of completeness.

Theorem 4. Suppose that $f$ is non-degenerate and that $n \geq 2$. Let (3) be the group of linear transformations of $\mathfrak{B}$ which keep $f$ invariant. Then the group of automorphisms of $\mathfrak{N}$ is isomorphic to (S. In case $k$ is the field of real numbers the Lie group (S) has for its Lie algebra the Lie algebra of derivations of $\mathfrak{\mathfrak { A }}$.

Proof. Let $\varphi$ be an automorphism of $\mathfrak{A}$. It is understood that $\varphi$ is a $k$-automorphism so that $\rho$ keeps scalar multiples of $e_{0}$ fixed. Suppose $\varphi e_{i}=\lambda_{i} e_{0}+v_{i}$ where $\lambda_{i} \in k, v_{i} \in \mathfrak{B}$ and $i=1,2, \cdots, n$. Then each product $\phi e_{i} \cdot \phi e_{j}=\mu_{i j} e_{0}+\lambda_{i} v_{j},+\lambda_{j} v_{i}, \mu_{i j} \in k$, must be a scalar multiple of $e_{0}$. Therefore $\lambda_{i} v_{j}+\lambda_{j} v_{i}=0$ and so $\varphi\left(\lambda_{j} e_{i}+\lambda_{i} e_{j}-2 \lambda_{i} \lambda_{j} e_{0}\right)=0$, which implies that $\lambda_{i}=\lambda_{j}=0$ if $i \neq j$. Therefore $\phi \mathfrak{B} \subseteq \mathfrak{B}$. Then $\varphi e_{i} \cdot \varphi e_{j}=$ $f\left(\varphi e_{i}, \phi e_{j}\right) e_{0}=\varphi\left(e_{i} \cdot e_{j}\right)=\varphi f\left(e_{i}, e_{j}\right) e_{0}=f\left(e_{i}, e_{j}\right) e_{0}$ for $i, j=1, \cdots, n$. Therefore $f\left(\varphi e_{i}, \varphi e_{j}\right)=f\left(e_{i}, e_{j}\right)$ for $i, j=1, \cdots, n$. Therefore the restriction of $\varphi$ to $\mathfrak{B}$ is an element of $(3)$. Conversely any element of (S) can be extended uniquely to an automorphism of $\mathfrak{A}$. Thus $\mathbb{B}$ is isomorphic to the group of automorphisms of $\mathfrak{A}$. Note that if these two groups are 
realized as groups of matrices with respect to the given basis, then the isomorphism is trivially birational and biregular in the sense of algebraic geometry, so that the groups are isomorphic as algebraic groups. The last statement of the theorem follows from a classical result in the theory of Lie groups (cf. [3] p. 137).

Theorem 5 (Wedderburn Principal Theorem). Suppose that $f$ is degenerate and either symmetric or alternating. Then $\mathfrak{A}$ has a semisimple subalgebra $\mathfrak{N}_{0}$ and a nilpotent ideal $\mathfrak{N}$ such that $\mathfrak{A}=\mathfrak{A}_{0}+\mathfrak{N}$ (vector space direct sum).

Proof. If $f$ is identically zero take $\mathfrak{N}=\mathfrak{B}$ and $\mathfrak{N}_{0}$ to be the subalgebra spanned by $e_{0}$. Otherwise let $\mathfrak{R}_{0}$ be the set of elements $u \in \mathfrak{B}$ such that $f(u, v)=0$ for all $v \in \mathfrak{B}$. Choose a basis $e_{1}, \cdots, e_{r+1}, \cdots, e_{n}$ for $\mathfrak{B}$ such that $e_{r+1} \cdots, e_{n} \operatorname{span} \mathfrak{R}_{0}$. Suppose first that $r \geqq 2$. Then $e_{0}, e_{1}, \cdots, e_{r}$ span a subalgebra $\mathfrak{A}_{0}$ which is isotopically left and right simple by Theorem 3. Taking $\mathfrak{N}=\mathfrak{N}_{0}$ it follows that $\mathfrak{A}=\mathfrak{U}_{0}+\mathfrak{N}$ with $\mathfrak{R}$ a nilpotent ideal of index two. Now suppose $r=1$. Then $e_{1}^{2}=\lambda e_{0}$ where $\lambda \neq 0$ in $k$. If the subalgebra spanned by $e_{0}$ and $e_{1}$ is semisimple, then $\mathfrak{A}_{0}$ and $\mathfrak{N}$ may be taken as before. Otherwise, suppose that $e_{0}+\beta e_{1}, \beta \neq 0$ in $k$, spans the one-dimensional radical of this subalgebra. Then take $\mathfrak{R}$ to be the ideal of $\mathfrak{Y}$ spannned by $e_{0}+\beta e_{1}, e_{2}$, $\cdots, e_{n}$ and $\mathfrak{\Re}_{0}$ to be the subalgebra spanned by $e_{0}$.

REMARK. The use of the terms "semisimple" and "nilpotent ideal" does not seem yet to be standardized in the literature on non-associative algebras. Although in the present case all of the customary interpretations of these terms are equivalent, nevertheless it desirable to give explicit definitions. An algebra is said to be semisimple if it is a direct sum of simple algebras, none of which is the zero algebra of dimension 1. An ideal is said to be nilpotent if there is an integer $m>0$ such that every product of $m$ elements of the ideal, irrespective of the manner of bracketing, is zero.

\section{REFERENCES}

1. A. A. Albert, Non-associative algebras I, Ann. of Math. 43 (1942), 685-707.

2. R. H. Bruck, Some results in the theory of linear non-associative algebras, Trans. Amer. Math. Soc. 56 (1944), 141-199.

3. C. C. Chevalley. Theory of Lie groups I, Princeton 1946.

BUCKNELL UNIVERSITY 


\section{PACIFIC JOURNAL OF MATHEMATICS}

\section{EDITORS}

David Gilbarg

Stanford University

Stanford, California

F. H. Brownell

University of Washington

Seattle 5, Washington
A. L. Whiteman

University of Southern Californıa Los Angeles 7, California

L. J. PaIge

University of California

Los Angeles 24, California

\section{ASSOCIATE EDITORS}
E. F. BECKENBACH
T. M. CHERRY
D. DERRY

E. HEWITT
A. HORN
L. NACHBIN
M. OHTSUKA

H. L. ROYDEN

M. M. SCHIFFER
E. SPANIER

E. G. STRAUS

F. WOLF

\section{SUPPORTING INSTITUTIONS}

\author{
UNIVERSITY OF BRITISH COLUMBIA \\ CALIFORNIA INSTITUTE OF TECHNOLOGY \\ UNIVERSITY OF CALIFORNIA \\ MONTANA STATE UNIVERSITY \\ UNIVERSITY OF NEVADA \\ NEW MEXICO STATE UNIVERSITY \\ OREGON STATE COLLEGE \\ UNIVERSITY OF OREGON \\ OSAKA UNIVERSITY \\ UNIVERSITY OF SOUTHERN CALIFORNIA
}

\author{
STANFORD UNIVERSITY \\ UNIVERSITY OF TOKYO \\ UNIVERSITY OF UTAH \\ WASHINGTON STATE COLLEGE \\ UNIVERSITY OF WASHINGTON \\ AMERICAN MATHEMATICAL SOCIETY \\ CALIFORNIA RESEARCH CORPORATION \\ HUGHES AIRCRAFT COMPANY \\ SPACE TECHNOLOGY LABORATORIES \\ NAVAL ORDNANCE TEST STATION
}

Mathematical papers intended for publication in the Pacific Journal of Mathematics should be typewritten (double spaced), and the author should keep a complete copy. Manuscripts may be sent to any one of the four editors. All other communications to the editors should be addressed to the managing editor, L. J. Paige at the University of California, Los Angeles 24, California.

50 reprints per author of each article are furnished free of charge; additional copies may be obtained at cost in multiples of 50 .

The Pacific Journal of Mathematics is published quarterly, in March, June, September, and December. The price per volume (4 numbers) is $\$ 12.00$; single issues, $\$ 3.50$. Back numbers are available. Special price to individual faculty members of supporting institutions and to individual members of the American Mathematical Society: $\$ 4.00$ per volume; single issues, $\$ 1.25$.

Subscriptions, orders for back numbers, and changes of address should be sent to Pacific Journal of Mathematics, 2120 Oxford Street, Berkeley 4, California.

Printed at Kokusai Bunken Insatsusha (International Academic Printing Co., Ltd.), No. 6, 2-chome, Fujimi-cho, Chiyoda-ku, Tokyo, Japan.

PUBLISHED BY PACIFIC JOURNAL OF MATHEMATICS, A NON-PROFIT CORPORATION

The Supporting Institutions listed above contribute to the cost of publication of this Journal, but they are not owners or publishers and have no responsibility for its content or policies. 


\section{Pacific Journal of Mathematics}

\section{Vol. 10, No. $2 \quad$ October, 1960}

Maynard G. Arsove, The Paley-Wiener theorem in metric linear spaces ........

Robert (Yisrael) John Aumann, Acceptable points in games of perfect

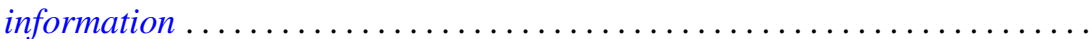

A. V. Balakrishnan, Fractional powers of closed operators and the semigroups generated by them ... . . . . . . . . . . . . . . . . . . . . . . . . . . . . 419

Dallas O. Banks, Bounds for the eigenvalues of some vibrating systems . . . . . 439

Billy Joe Boyer, On the summability of derived Fourier series . . . . . . . . . . . 475

Robert Breusch, An elementary proof of the prime number theorem with

remainder term ...................................

Edward David Callender, Jr., Hölder continuity of $n$-dimensional

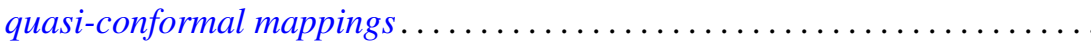

L. Carlitz, Note on Alder's polynomials ......................... 517

P. H. Doyle, III, Unions of cell pairs in $E^{3} \ldots \ldots \ldots \ldots \ldots \ldots \ldots \ldots \ldots \ldots \ldots \ldots . \ldots 21$

James Eells, Jr., A class of smooth bundles over a manifold . . . . . . . . . . . . 525

Shaul Foguel, Computations of the multiplicity function . . . . . . . . . . . . . . 539

James G. Glimm and Richard Vincent Kadison, Unitary operators in

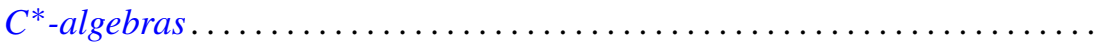

Hugh Gordon, Measure defined by abstract $L_{p}$ spaces . . . . . . . . . . . 557

Robert Clarke James, Separable conjugate spaces ....................

William Elliott Jenner, On non-associative algebras associated with bilinear forms

Harold H. Johnson, Terminating prolongation procedures

John W. Milnor and Edwin Spanier, Two remarks on fiber homotopy type .

Donald Alan Norton, A note on associativity . .

Ronald John Nunke, On the extensions of a torsion module.

Joseph J. Rotman, Mixed modules over valuations rings . . . . .

A. Sade, Théorie des systèmes demosiens de groupoï des . .

Wolfgang M. Schmidt, On normal numbers . .

661

Berthold Schweizer, Abe Sklar and Edward Oakley Thorp, The metrization of

statistical metric spaces

John P. Shanahan, On uniqueness questions for hyperbolic differential

equations

A. H. Stone, Sequences of coverings

Edward Oakley Thorp, Projections onto the subspace of compact operators

L. Bruce Treybig, Concerning certain locally peripherally separable spaces

Milo Wesley Weaver, On the commutativity of a correspondence and a

permutation

David Van Vranken Wend, On the zeros of solutions of some linear complex

differential equations. 